\title{
Highly Proliferative Fibroblasts Forming Fibrotic Focus Govern Metastasis of Invasive Ductal Carcinoma of the Breast
}

Takahiro Hasebe, M.D., Satoshi Sasaki, M.D., Shigeru Imoto, M.D., Atsushi Ochiai, M.D.

Pathology Division (TH, AO); Epidemiology and Biostatistics Division (SS), National Cancer Center

Research Institute East; and Department of Surgery (SI), National Cancer Center Hospital East, Kashiwa,

Chiba, Japan

We have already reported that invasive ductal carcinomas (IDCs) with fibrotic focus (FF) have more aggressive characteristics than those without FF. FF is composed of a mixture of fibroblasts and various amounts of collagen fibers, suggesting that highly proliferative fibroblasts forming FF increase the malignant potential of IDCs with FF. The purpose of this study was to examine whether there is a difference of proliferative activity of fibroblasts forming and not forming FF, which plays an important role in the tumor progression of IDCs. Two hundred three consecutive cases of IDC of the breast surgically treated at the National Cancer Center Hospital East formed the basis for this study. The proliferative activity of the fibroblasts forming the FF was immunohistochemically evaluated by using mouse MIB-1 monoclonal antibody against Ki-67 antigen. The MIB-1 labeling index (LI) is the percentage of fibroblasts forming FF that have positively stained nuclei, and 300 fibroblasts were counted in each FF. The significance of the proliferative activity of fibroblasts forming FF with regard to lymph node metastasis (LNM) or distant-organ metastasis (DOM) was compared with well-known prognostic parameters. Multivariate analysis demonstrated that high MIB-1 LI of fibroblasts forming FF significantly increased the relative risk of $L N M$ and the hazard rate of DOM $(P<.001$ and $P=.009)$. The present study indicated that the metastatic ability of IDCs with FF

Copyright (C) 2001 by The United States and Canadian Academy of Pathology, Inc.

VOL. 14, NO. 4, P. 325, 2001 Printed in the U.S.A.

Date of acceptance: September 8, 2000.

This work was supported in part by a Grant-in-Aid for Cancer Research from the Ministry of Health and Welfare and by a Grant-in-Aid for the Second Term Comprehensive 10-Year Strategy for Cancer Control from the Ministry of Health and Welfare in Japan.

Part of this work was presented at the 89th annual meeting of the Japanese Society of Pathology on April 13, 2000 in Osaka, Japan.

Address reprint requests to: Atsushi Ochiai, M.D., Pathology Division, National Cancer Center Research Institute East, Kashiwanoha 6-5-1, Kashiwa, Chiba 277-8577, Japan; e-mail: aochiai@east.ncc.go.jp; fax: +81-471-34-6865. is highly dependent on the proliferative activity of the fibroblasts forming FF.

KEY WORDS: Angiogenesis, Fibrotic focus, Fibroblast, Immunohistochemistry, Invasive ductal carcinoma, Ki-67 antigen, Prognosis.

Mod Pathol 2001;14(4):325-337

We have already reported that invasive ductal carcinomas (IDCs) with fibrotic focus (FF) have more aggressive characteristics than IDCs without FF (1) and that the former are associated with significantly poorer survival course in short- and long-term survival periods than the latter $(2,3)$. We also found that IDCs with FF exhibit significantly greater tumor angiogenesis and higher tumor cell proliferative activity and that the presence of FF is an independent prognostic parameter for IDC patients (4). These findings indicate that the presence of FF in IDCs is a very important histological parameter for predicting the outcome of patients with IDC of the breast.

FF is composed of a mixture of fibroblasts and various amounts of collagen fibers, and they occupy almost the entire center of the IDC. In routine examination, mitotic figures of fibroblasts forming FF as well as those of the tumor cells are seen in some areas within FF. This suggests that highly proliferative fibroblasts make up FF and heighten the malignant potential of IDCs having FF.

The purposes of this study were to determine whether there are biological characteristic differences between fibroblasts forming and not forming FF by evaluating their proliferative activity and also whether their proliferative activity plays a important role in tumor progression of IDC. The significance of the proliferative activity of fibroblasts forming and not forming FF on lymph node metastasis (LNM) and distant-organ metastasis (DOM) in IDC patients was assessed by comparing it with well-known histological parameters, tumor cell 
proliferative activity, and tumor angiogenesis, which are significant prognostic factors for breast cancer patients (5-7). The present study demonstrated that the proliferative activity of the fibroblasts forming FF is an independent parameter predicting LNM and DOM by IDCs with FF and that it was also an important prognostic parameter for IDCs without FF. The results indicated that the proliferative activity of intratumoral fibroblasts is a universal prognostic parameter for IDCs with and without FF.

\section{MATERIALS AND METHODS}

\section{Cases}

Two hundred four consecutive cases of IDC of the breast surgically treated between July 1992 and November 1996 at the National Cancer Center Hospital East constituted the basis of this study. Clinical information was obtained from the patients' medical records. All of the patients were Japanese women ranging in age from 28 to 87 years old (average, $53 \mathrm{y}$ ), and all had a solitary lesion. One hundred five patients were premenopausal, and 101 were postmenopausal. Standard radical mastectomy in 170, and quadrantectomy in 19. Axillary lymph node dissection was carried out in 196 patients. None of the patients had received radiotherapy or chemotherapy before surgery. All tumors were classified according to the pathological TNM (pTNM) classification (8).

For pathological examination, the surgically resected specimens were fixed in $10 \%$ formalin overnight at $4^{\circ} \mathrm{C}$, and the entire tumor was cut into slices at intervals of 0.5 to $0.7 \mathrm{~cm}$. The size and gross appearance of the tumors were recorded, and the former was validated by comparison with tumor size on histological slides. Multiple histological sections were taken from each tumor to measure the maximum tumor diameter and area. The sections were processed routinely and embedded in paraffin.

\section{Histological Examination}

Serial sections of each tumor area were cut from the paraffin blocks. One section was stained with hematoxylin and eosin and examined pathologically to confirm the diagnosis, and the remaining sections were used for immunohistochemistry. All tumors were classified according to the guidelines of the World Health Organization (9), and their histologic grade was evaluated by the classification of Elston (10).

\section{Histological Features of FF}

FF is defined according to the following characteristics (1-4):
- FF often consists of fibrous bands radially expanding into the surrounding area and with the appearance of a scar (Fig. 1A) or a radiating fibrosclerotic core (Fig. 1C). Some FFs have an irregular, moth-eaten appearance (Fig. 1E). FFs are located at almost the exact center of the tumor. They are surrounded by a more cellular zone of infiltrating ductal carcinoma cells and occupy various percentages of the tumor area (Fig. 1, A, C, and E).

- Tumor cells are seldom seen in FFs $3 \mathrm{~mm}$ or less in size (Fig. 2). However, tumor cells growing in a scirrhous fashion or in solid nests are observed in FFs as their size increases (Fig. 1, $\mathrm{B}, \mathrm{D}$, and F).

- The fibroblasts and collagen fibers in FFs are arranged in irregular or storiform patterns with increased fibroblasts cellularity and/or collagenization (Fig. 1, B, D, and F). The degree of fibrosis in FFs is classified into three grades according to the following criteria: Grade 1, a large number of fibroblasts with small amount of collagen fibers (Fig. 3A); Grade 3, mainly composed of collagen fibers, mostly hyalinized (Fig. 3C); and Grade 2, intermediate between Grades 1 and 3, with fibroblasts and collagen fibers intermingled in various ratios (Fig. 3B). The arrangement of fibroblasts or collagen fibers forming FFs differs from that of the surrounding tumor stroma, which is more orderly, or from that of the normal breast tissue stroma. The difference in arrangement of the fibroblasts is the characteristic that most distinguishes fibroblasts forming FFs from those outside FFs. Some FFs are associated with coagulation necrosis of the tumor. The area of tumor cell coagulation necrosis within FF is smaller than that occupied by the fibroblasts or collagen fibers. Coagulation necrosis within tumors without accompanying fibroblasts or collagen fiber proliferation is insufficient to be called FF. Whenever several FFs of various sizes were present in the same tumor, the characteristics of the largest one were used in the study. In addition, a diameter of $1 \mathrm{~mm}$ or more is required for a fibrosclerotic core to be considered FF in IDC.

\section{Immunohistochemistry}

Immunohistochemical staining was performed by the avidin-biotin-peroxidase complex technique (11). The primary antibodies employed were an affinity-purified monoclonal antibody against estrogen receptor (ER) protein (1D5, DAKO, Grostrup, Denmark) used at a 1:100 dilution; polyclonal antibody specific for the $185-\mathrm{kDa}$ c-erbB-2 protein (Nichirei, Tokyo, Japan) used at a 1:200 dilution; a 


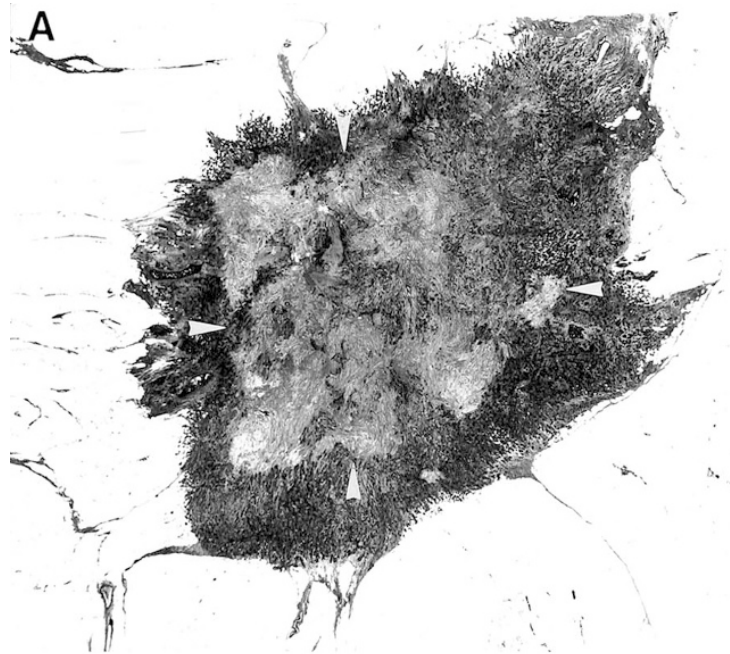

C

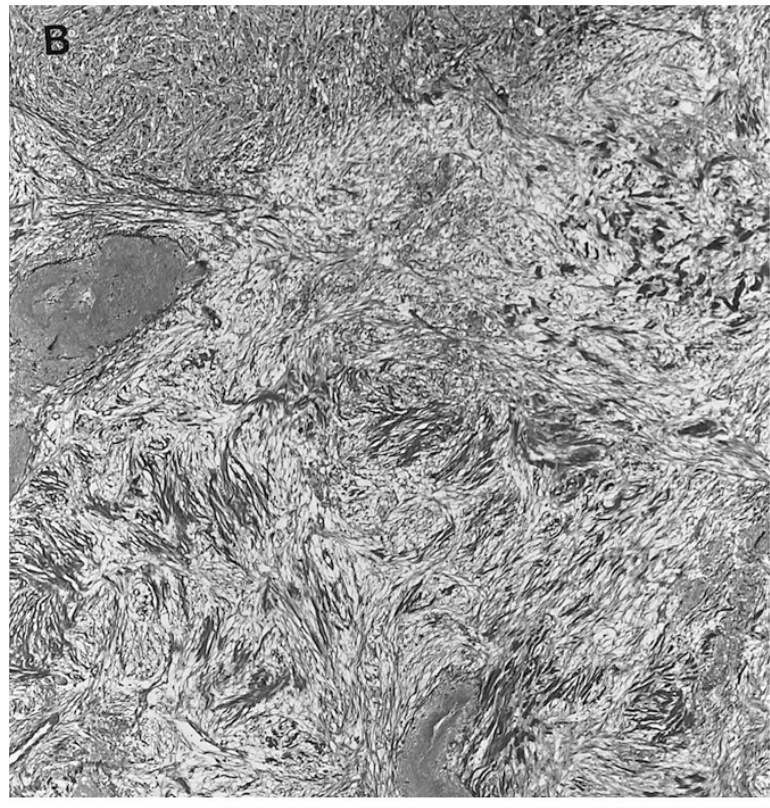

$\mathrm{E}$
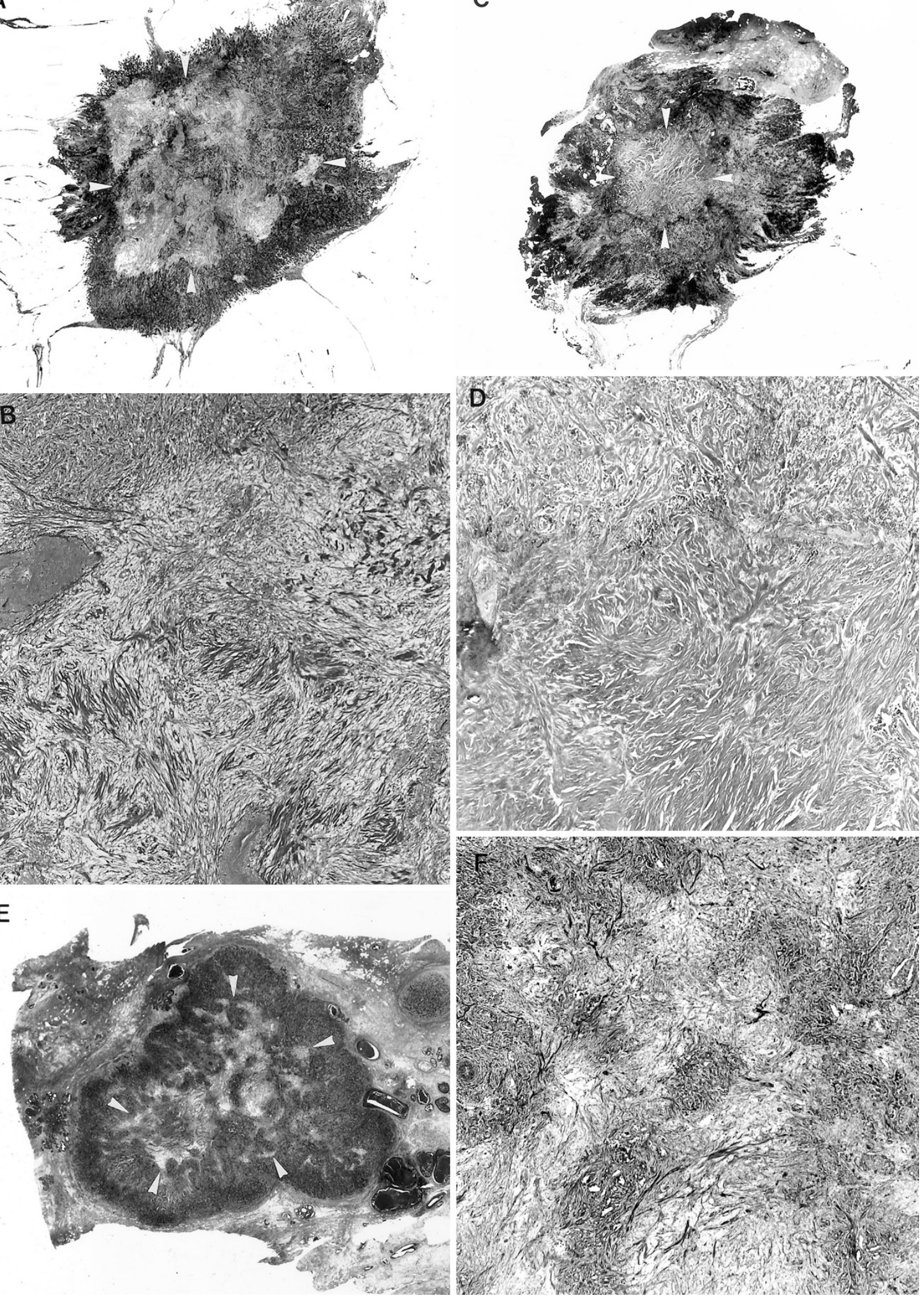

FIGURE 1. Invasive ductal carcinomas with fibrotic focus (FF). A, An FF measuring $13 \times 10$ mm in size is observed within the tumor (arrowheads). The FF shows scar-like features and is surrounded by invasive ductal carcinoma cells. Some residual tumor islands of various sizes are observed within the FF (panoramic view). B, The fibroblasts and collagen fibers composing the FF show a storiform arrangement, and some vessels showing elastoid degeneration of their wall are seen within the FF (original magnification, $20 \times$ ). C, An FF measuring $5 \times 7$ mm in size is observed within the tumor (arrowheads). The FF has the appearance of a radiating fibrosclerotic core (panoramic view). D, The FF consists of hyalinized collagen fibers admixed with small amounts of fibroblasts. The collagen fibers show a storiform pattern (original magnification, $20 \times$ ). E, An FF measuring $10 \times 8 \mathrm{~mm}$ in size is observed within the tumor (arrowheads). The FF has an irregularly moth-eaten appearance and contains several residual tumor islands of various sizes (panoramic view). F, Residual tumor islands blend with fibroblasts or collagen fibers arranged in a storiform pattern (original magnification, $20 \times$ ). 

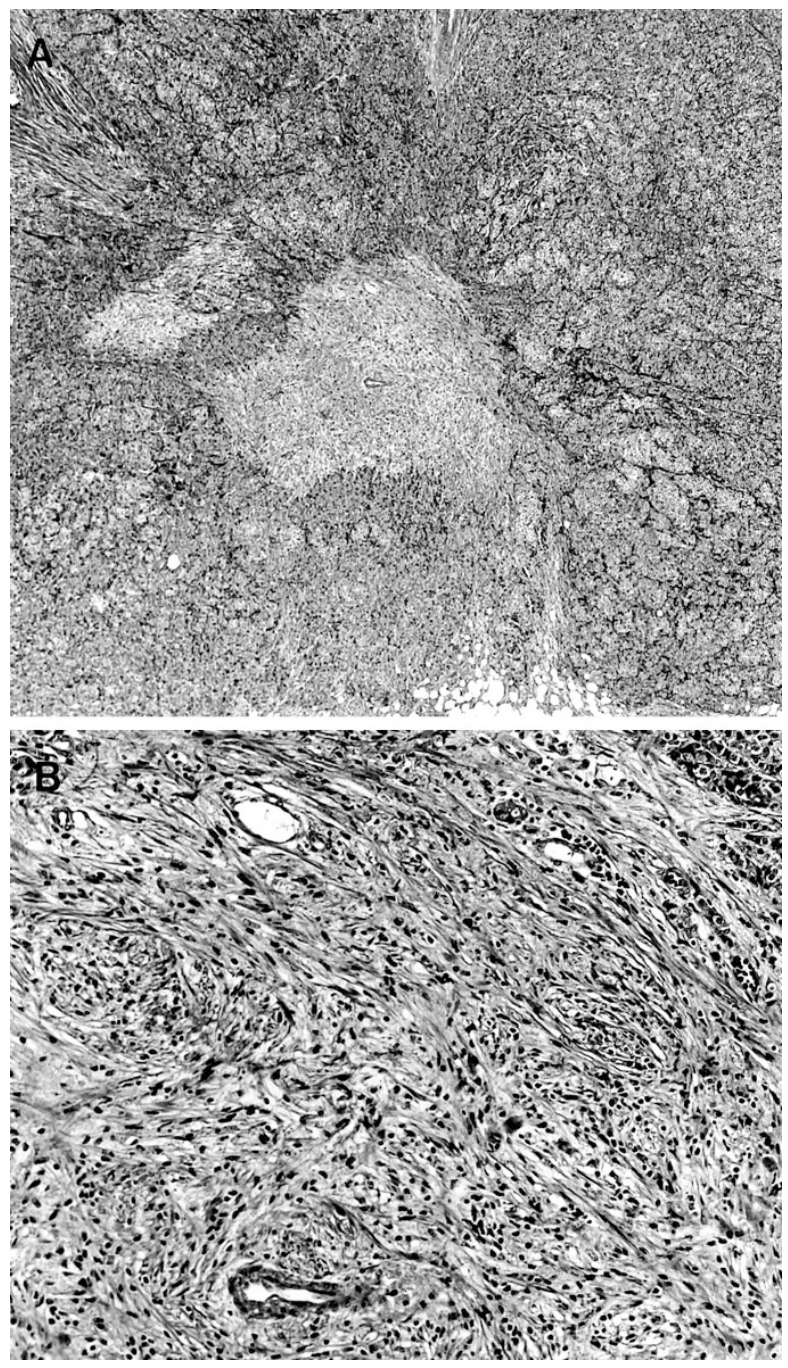

FIGURE 2. A, A small fibrotic focus (FF) measuring $2 \times 2 \mathrm{~mm}$ in size is observed within the tumor (original magnification, $20 \times$ ). B, The fibroblasts forming the FF are arranged in a storiform pattern and are intermingled with inflammatory cells (original magnification, 100×).

mouse monoclonal antibody against Ki-67 antigen (MIB-1, Immunotech, Marseille, France), applied at a 1:50 dilution; and a mouse monoclonal antibody against human endothelial cells (CD31, JC/70A, DAKO), used at a 1:50 dilution. Microwave treatment was performed before immunohistochemical staining for MIB-1 (12). Sections were treated with $0.05 \%$ pepsin before staining for CD31. After immunostaining, the sections were counterstained with hematoxylin and eosin. Sections of IDC positive for ER protein, c-erbB-2 protein, MIB-1, and CD31 were used each time as a positive control, and the primary antibody was replaced with normal rabbit serum or normal mouse immunoglobulin as a negative control.

\section{Assessment of the Immunohistochemistry Findings}

Tumor cell nuclei stained brown to dark brown were considered positive for ER or MIB-1, and
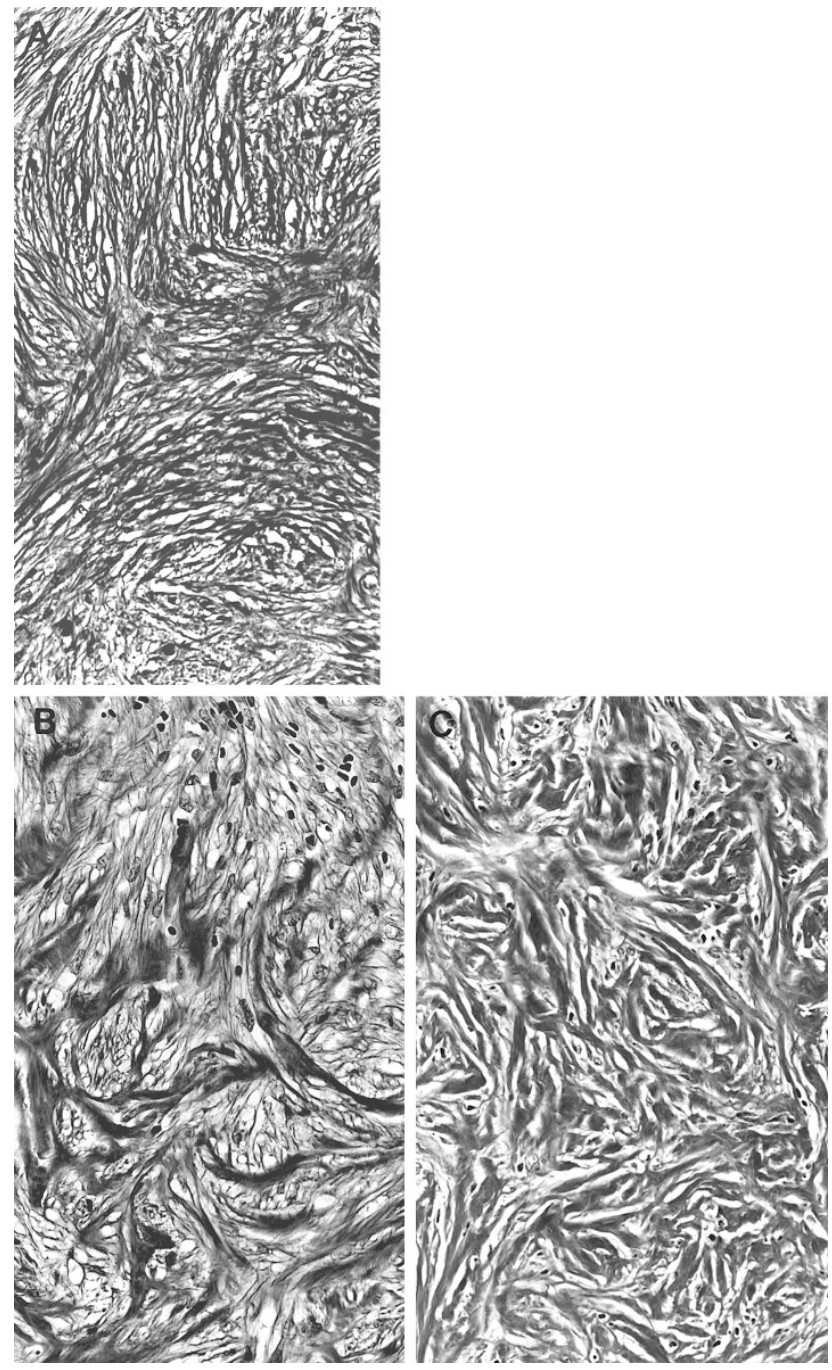

FIGURE 3. Fibrosis grade of fibrotic focus (FF; original magnification, $100 \times)$. A, Grade l. FF consists only of fibroblasts arranged in a storiform pattern. B, Grade 2. FF consists of fibroblasts and hyalinized collagen fibers showing a storiform arrangement. C, Grade 3. FF consists mainly of hyalinized collagen fibers showing a storiform pattern.

faintly stained nuclei were considered negative. Nuclear staining for ER was only considered positive when more than $10 \%$ of the tumor cells in the entire tumor area were judged to be positive. Tumors were judged to be positive for c-erbB-2 protein when the cell membrane of more than $10 \%$ of the tumor cells throughout the tumor stained positive.

The MIB-1 labeling index (LI) is the ratio of tumor cells or fibroblasts with positively stained nuclei to the total number of tumor cells or fibroblasts counted, expressed as a percentage. We previously demonstrated that the proliferative activity of tumor cells or microvessel counts (MVC) is significantly higher in the outer area of IDCs than in the inner area (4), and for that reason, the fields for cell counting to assess the proliferative activity of tumor cells or for MVC were selected in the outer area of 
the IDCs (Fig. 4). The inner area was defined as a circle having a radius half that of the radius of the tumor, and the outer area was defined as the zone surrounding the inner area of the tumor. At least 500 tumor cells in the outer area were counted in each high-power field $(400 \times)$ in each IDC. The MVCs were made by identifying the field with the highest neovascularity in the outer area of the tumor by scanning the tumor sections stained for CD31 at low-power magnification $(40 \times$ and $100 \times$ total magnification; Fig. 4) and then counting the individual microvessels within $200 \times$ fields $(20 \times$ objective and $10 \times$ ocular; $0.384 \mathrm{~mm}^{2}$ per field). Brown-staining endothelial cell clusters consisting of two or more cells with formation of a definite lumen that were clearly separated from the adjacent microvessels, tumor cells, and other connective tissue elements were considered to be a single, countable microvessel. Lumina without a rim of CD31-positive cells were not counted, even if they contained blood cells. Results are expressed as the highest number of microvessels in any single $200 \times$ fields $(4,13)$.

The MIB-1 LI (\%) of fibroblasts was determined by counting a total of 300 fibroblasts in the inner and outer areas in each high-power field, and 600 fibroblasts within the tumor were counted in each IDC without FF. The MIB-1 LI of fibroblasts forming and not forming FF in IDCs with FF was assessed. If IDCs had FF of small size, such as $3 \mathrm{~mm}$ in size, and the number of fibroblasts forming the FF was inadequate to calculate the MIB-1 LI (\%) of the fibroblasts forming the FF, the MIB-1 LI of fibroblasts was calculated in the fibroblasts surrounding the FF as well as in those forming FF.

Two of the authors (TH and $\mathrm{AO}$ ) determined the MIB-1 LIs of the tumor cells and fibroblasts and the MVCs. If the MIB-1 LI or MVC values counted by $\mathrm{TH}$ and $\mathrm{AO}$ were almost the same, the values obtained by TH were used in this study. However, if they were not almost the same, we reexamined them to come to an agreement.

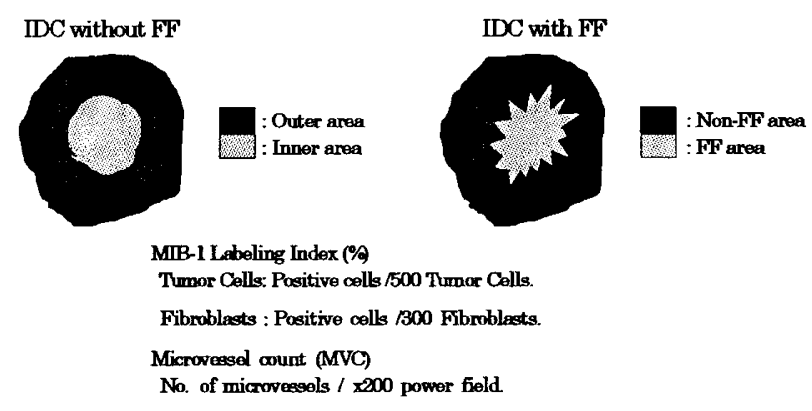

FIGURE 4. Schematic drawing of assessments of the MIB-1 labeling indexes of the tumor cells and fibroblasts and of the microvessel counts of invasive ductal carcinomas with and without fibrotic focus.

\section{Outcome}

The survival of the patients was evaluated by follow-up for a median period of 41 months, to June of 1999. One hundred fifty-nine patients were alive and well, 47 had tumor recurrence, 29 had DOM, and 22 had died of their disease. Among the patients with DOM, 24 patients had IDCs with FF, and 18 of the 22 patients who also died of their disease had IDCs with FF. Measurements of DOM-free survival and overall survival (OS) rates started at the time of surgery. Tumor relapse was considered to have occurred whenever there was evidence of metastasis or local recurrence. DOM was observed in the following organs: bone, 12 cases; liver, 9 cases; lung, 6 cases; and brain, 2 cases. Only deaths due to breast cancer were considered for the purpose of this study, and all of the patients who died of the disease had DOM.

\section{Statistical Analysis}

We analyzed the predictive power of the presence of FF for LNM, DOM, and OS by multivariate analyses using the logistic regression model (14) and by the Cox proportional hazard regression model (15) using the step-down method until all of the remaining factors were significant at a $P$ value below .05 . For the comparative study with the presence of FF for LNM, DOM, or OS, the following variables were examined as potential predictive parameters for LNM or DOM: invasive tumor size $(\leq 20 v s$. more than $20 \mathrm{~mm}$ ), histological grade (1 or $2 v s .3$ ); tumor necrosis (absent vs. present); lymphatic invasion (absent $v s$. present); vascular invasion (absent $v$ s. present); adipose tissue invasion (absent $v s$. present); nodal status (negative $v s$. positive); ER expression (negative $v s$. positive); and c-erbB-2 expression (negative $v s$. positive). The parameters that showed a significant correlation with LNM in the univariate analyses were then entered into the multivariate analyses.

In the cases as a whole, the differences between the proliferative activity of the tumor cells and of the fibroblasts of IDCs with and without FF were evaluated by the $t$ test. Similarly, the difference between the MVCs of IDCs with and without FF was also examined by the $t$ test. Pearson's correlations between the MIB-1 LIs of the tumor cells, the MIB-1 LIs of the fibroblasts, and the MVCs were also separately examined by linear correlation in IDCs with and without FF.

Associations between the presence of FF and other parameters were examined by the $\chi^{2}$ test. The median values of the proliferative activity of tumor cells and of the fibroblasts of the IDCs as a whole were used as the cutoff values predicting the presence of FF. Similarly, the median cutoff values of the MVCs of all IDCs were used for predicting the 
presence of FF. MIB-1 LI and MVC values equal to or below the median value were considered to be low MIB-1 LI or low MVC values, and MIB-1 LI and MVC values higher than the median values, to be high MIB-1 LI or high MVC values. All parameters showing significant correlations with the presence of FF were entered into multivariate analysis by using the step-down logistic regression model to identify the parameter that was most significantly associated with the presence of FF.

To identify the parameter with the strongest effect on LNM, DOM, and OS in IDCs with FF, multivariate analyses were performed for parameters significantly correlated with the presence of FF in the multivariate analysis, fibrosis grade, and diameter of FF.

All analyses were performed with Statistica/Windows software (StatSoft, Tulsa, OK).

\section{RESULTS}

\section{Effect of FF on LNM, DOM, and OS}

Univariate analyses for LNM showed that parameters significantly associated with LNM were the presence of FF, lymphatic invasion, vascular invasion, invasive tumor size of $20 \mathrm{~mm}$ or more, c-erbB-2 expression, and adipose tissue invasion $(P$ $<.001, P<.001, P=.018, P<.001, P=.025$, and $P=.029$ ). There were no significant associations between LNM and tumor necrosis, histological grade, or ER status in the univariate analyses $(P=$ $.236, P=.082$, and $P=.133$. Multivariate analysis clearly showed that the presence of FF, lymphatic invasion, and invasive tumor size of $20 \mathrm{~mm}$ or more significantly increased the relative risks (RRs) of LNM (Table 1). Vascular invasion, c-erbB-2 expression, and adipose tissue invasion failed to significantly increase the RRs of LNM.

Parameters significantly associated with DOM in the univariate analyses were the presence of $\mathrm{FF}$, high histological grade, LNM, ER-negative status, invasive tumor size of $20 \mathrm{~mm}$ or more, and adjuvant therapy $(P<.001, P<.001, P=.002, P=.015$, and $P=.005$, respectively). The presence of FF, high histological grade, and the presence of LNM significantly increased the hazard rates (HRs) of DOM in the multivariate analysis (Table 1), whereas ER status, invasive tumor size, and adjuvant therapy failed to significantly increase the HR of DOM. There were no significant correlations between DOM and tumor necrosis, lymphatic invasion, vascular invasion, c-erbB-2 expression, or adipose tissue invasion in the univariate analyses $(P=.236, P$ $=.632, P=.290, P=.207$, and $P=.937$, respectively).

In the univariate analyses for OS, the presence of FF, high histological grade, LNM, ER-negative sta- tus, invasive tumor size of $20 \mathrm{~mm}$ or more, and adjuvant therapy showed significant correlations with OS $(P=.002, P<.001, P<.001, P<.001, P=$ .017 , and $P=.013$, respectively), and the presence of FF, high histological grade, and the presence of LNM significantly increased the HRs of OS (Table 1). ER status, invasive tumor size, and adjuvant therapy, however, failed to significantly increase the HR of OS. The presence of tumor necrosis, vascular invasion, lymphatic invasion or c-erbB-2 expression, however, showed no significant correlation with $\mathrm{OS}$ in the univariate analyses $(P=.313$, $P=.321, P=.073$, and $P=.079$, respectively).

Differences in the Proliferative Activity of Tumor Cells, the Proliferative Activity of Intratumoral Fibroblasts, and the MVCs Between IDCs With and Without FF

The mean MIB-1 LI (\%) of the tumor cells in IDCs with FF was $41.1 \pm 16.9$, and the LI of those in IDCs without FF was $31.6 \pm 15.4$. The former showed a significantly higher tumor cell MIB-1 LI (\%) than the latter $(P<.001)$. Similarly, the former had a significantly higher mean MVC $(46.5 \pm 31.0)$ than the latter $(35.7 \pm 20.3 ; P=.002)$.

The mean MIB-1 LIs (\%) of the fibroblasts in the inner and outer areas of IDCs without FF were almost the same (inner area, $4.9 \pm 3.6$; outer area, $4.9 \pm 3.4$ ). By contrast, in IDCs with FF, the mean MIB-1 LI (\%) of the fibroblasts forming FF was 10.7 \pm 7.2 , and that of those not forming FF was $5.9 \pm$ 5.2. The former had a significantly higher MIB-1 LI (\%) than the latter $(P<.001$, Fig. 5). The mean MIB-1 LI (\%) of the fibroblasts forming FF was also significantly higher than the mean MIB-1 LIs of the fibroblasts in the inner and outer areas of IDCs without FF $(P<.001)$.

\section{Correlations Between the Proliferative Activity of} Tumor Cells, the Proliferative Activity of Intratumoral Fibroblasts, and the MVCs of IDCs With and Without FF

In IDCs with FF the MIB-1 LIs of the tumor cells were significantly correlated with those of the fibroblasts forming FF and those not forming FF (Table 2). Although MVCs were significantly correlated with the MIB-1 LIs of the fibroblasts not forming $\mathrm{FF}$, there was no significant association between the MVC and the MIB I LIs of the tumor cells or that of the fibroblasts forming FF. The diameter of FF was a significantly correlated with the MVCs, but no significant association was observed between the diameter of FF and the MIB-1 LIs of the tumor cells or fibroblasts. FF grade was also significantly correlated with the MVCs and significantly negatively correlated with the MIB-1 tumor cell LI. There was 
TABLE 1. Histological Parameters Significantly Associated with LNM, DOM, and OS in IDC

\begin{tabular}{|c|c|c|c|c|c|}
\hline \multirow{2}{*}{ Parameters } & \multirow{2}{*}{ Total } & \multicolumn{4}{|c|}{ Multivariate for LNM } \\
\hline & & LNMR (\%) & RR & $95 \%$ CI & $P$ value \\
\hline All cases & 196 & $104(53)$ & & & \\
\hline \multicolumn{6}{|l|}{ Fibrotic focus } \\
\hline Absent & 94 & $38(40)$ & Referent & & \\
\hline Present & 102 & $66(65)$ & 2.3 & $1.2-6.1$ & .011 \\
\hline \multicolumn{6}{|l|}{ Lymphatic invasion } \\
\hline Absent & 106 & $41(39)$ & Referent & & \\
\hline Present & 90 & $63(70)$ & 3.9 & $1.7-6.1$ & $<.001$ \\
\hline \multicolumn{6}{|c|}{ Invasive tumor size $(\mathrm{mm})$} \\
\hline$\leq 20$ & 72 & $26(36)$ & Referent & & \\
\hline$>20$ & 124 & $78(63)$ & 2.0 & $1.0-3.8$ & .045 \\
\hline \multicolumn{6}{|l|}{ c-erbB-2 expression } \\
\hline Negative & 115 & $53(46)$ & Referent & & \\
\hline Positive & 81 & $51(63)$ & & & \\
\hline \multicolumn{6}{|l|}{ Vascular invasion } \\
\hline Absent & 149 & $72(48)$ & Referent & & \\
\hline Present & 47 & $32(68)$ & & & \\
\hline \multicolumn{6}{|c|}{ Adipose tissue invasion } \\
\hline Absent & 20 & $6(30)$ & Referent & & \\
\hline \multirow[t]{3}{*}{ Present } & 176 & $98(56)$ & & & \\
\hline & & \multicolumn{4}{|c|}{ Multivariate for DOM } \\
\hline & & DOMR (\%) & HR & $95 \% \mathrm{CI}$ & $P$ value \\
\hline All cases & 204 & $29(14)$ & & & \\
\hline \multicolumn{6}{|l|}{ Fibrotic focus } \\
\hline Absent & 99 & $5(5)$ & Referent & & \\
\hline Present & 105 & $24(23)$ & 3.4 & $1.3-9.0$ & .014 \\
\hline \multicolumn{6}{|l|}{ Histological grade } \\
\hline 1 and 2 & 142 & $10(7)$ & Referent & & \\
\hline 3 & 62 & $19(31)$ & 4.5 & $2.1-9.9$ & .001 \\
\hline \multicolumn{6}{|l|}{ Nodal status } \\
\hline Absent & 92 & $4(14)$ & Referent & & \\
\hline Present & 104 & $24(23)$ & 5.1 & $1.8-14.9$ & .003 \\
\hline \multicolumn{6}{|l|}{ ER status } \\
\hline Negative & 59 & $15(25)$ & Referent & & \\
\hline Positive & 145 & $14(10)$ & & & \\
\hline Invasive tumor size & & & & & \\
\hline$\leq 20$ & 74 & $5(7)$ & Referent & & \\
\hline$>20$ & 130 & $24(19)$ & & & \\
\hline Adjuvant therapy & & & & & \\
\hline No & 55 & $2(4)$ & Referent & & \\
\hline Yes & 149 & $27(18)$ & & & \\
\hline & & & Multi & & \\
\hline & & MR (\%) & HR & $95 \% \mathrm{CI}$ & $P$ value \\
\hline All cases & 204 & $22(11)$ & & & \\
\hline Fibrotic focus & & & & & \\
\hline Absent & 99 & $4(4)$ & Referent & & \\
\hline Present & 105 & $18(17)$ & 3.0 & $1.0-8.9$ & .045 \\
\hline Histological grade & & & & & \\
\hline 1 and 2 & 142 & $3(2)$ & Referent & & \\
\hline 3 & 62 & $19(31)$ & 13.5 & $4.1-46.8$ & $<.001$ \\
\hline Nodal status & & & & & \\
\hline Absent & 92 & $3(3)$ & Referent & & \\
\hline Present & 104 & 19 (18) & 4.3 & $1.3-14.4$ & .020 \\
\hline ER status & & & & & \\
\hline Negative & 59 & $14(24)$ & Referent & & \\
\hline Positive & 145 & $8(5)$ & & & \\
\hline Invasive tumor size & & & & & \\
\hline$\leq 20$ & 74 & $3(4)$ & Referent & & \\
\hline$>20$ & 130 & $19(15)$ & & & \\
\hline Adjuvant therapy & & & & & \\
\hline No & 55 & $1(2)$ & Referent & & \\
\hline Yes & 149 & $27(18)$ & & & \\
\hline
\end{tabular}

In order to identify the parameter with the strongest effect on LNM, DOM, and OS in IDCs with FF, multivariate analyses were performed for parameters significantly correlated with the presence of FF in the multivariate analysis, fibrosis grade and diameter of FF. The multivariate analyses for LNM were performed by the logistic regression model, and those for DOM and MR were performed by the Cox proportional hazard regression model. The multivariate analyses were adjusted with parameters that were significantly correlated with LNM, DOM, or OS listed on the table using the step-down method until all the remaining factors were significant at a $P$ level below 0.05 .

OS, overall survival; LNM, lymph node metastasis; LNMR, LNM rate; RR, relative risk; CI, confidence interval; DOM, distant organ metastasis; DOMR, DOM rate; MR, mortality rate; IDC, invasive ductal carcinoma; HR, hazard rate; ER, estrogen receptor. 


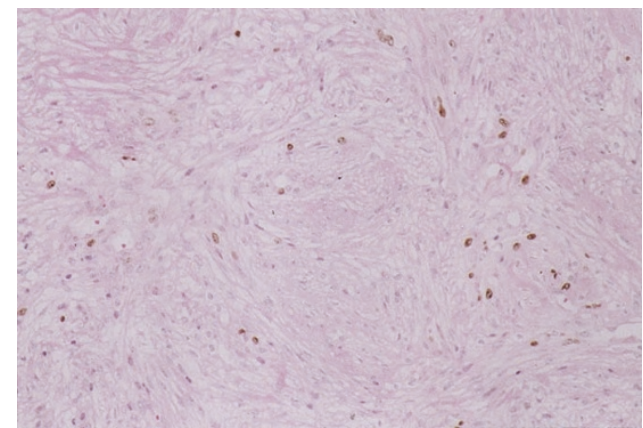

FIGURE 5. Immunohistochemical staining for MIB-1 in the fibroblasts forming fibrotic focus within IDC. Several fibroblasts show positive nuclear staining for MIB-1 (original magnification, 100×)

no significant correlation between the FF grades and the MIB-LI of the fibroblasts.

In IDCs without FF, the MIB-1 LIs of the tumor cells were significantly correlated with the MIB-LIs of the fibroblasts in both the inner and outer area $(r$ $=0.29, P<.01$; and $r=0.39, P<.01$, respectively), but there was no significant correlation between the MIB-LIs of the tumor cells and the MVCs $(r=$ $0.09, P=.40$ ). There was a significant correlation between the MIB-1 LIs of the fibroblasts in the inner area and in the outer area $(r=0.57, P<.01)$. Although the MIB-1 LIs of fibroblasts in the outer area were significantly associated with the MVCs ( $r$ $=0.22, P=.03)$, the LIs of those in the inner area failed to show a significant correlation with the MVCs $(r=0.18, P=.07)$.

\section{Multivariate Analysis for the Presence of FF}

The cutoff value of the MIB-1 LIs of tumor cells was set at 33\%, based on the median MIB-1 LI of the IDCs. Tumors that had an MIB-1 LI of tumor cells or fibroblasts in the inner or outer area equal to or below the cutoff value were classified into the lowMIB-1 LI group. All other tumors, that is, those with MIB-1 LI values above the cutoff value, formed the high-MIB-1 LI group. The cutoff value of MVC was set at $37 \%$, based on the median MVCs of all IDCs in both areas. Tumors with an MVC at or below the cutoff value were classified into the low MVC group, and those an MVC greater than the cutoff value formed the high MVC group.

Among the parameters significantly associated with the presence of FF in the univariate analyses, the MIB-1 LIs of the tumor cells, invasive tumor size, lymph node metastasis, and the MVC still significantly increased the RRs of the presence of FF in the multivariate analysis (Table 3). Other parameters failed to increase the RR of the presence of FF in multivariate analysis. Lymphatic invasion, c-erbB-2 expression, and the MIB-1 LIs of the fibroblasts in the outer area were not significantly correlated with the presence of $\mathrm{FF}$ in the univariate analyses $(P=.245, P=.175$, and $P=.549$, respectively).

\section{Multivariate Analyses for LNM, DOM, and OS in} IDCs with FF

Univariate and multivariate analyses for LNM, DOM, and OS were performed by parameters significantly associated with the presence of FF, the proliferative activity of the fibroblasts forming and not forming FF, fibrosis grade and diameter of FF, and adjuvant therapy status. The cutoff values of the MIB-1 LIs of the fibroblasts forming and not forming $\mathrm{FF}$ were set at $10 \%$ and $5 \%$, respectively, based on the median MIB-1 LI of IDCs with FF. Tumors which had the MIB-1 LIs of the fibroblasts forming or not forming $\mathrm{FF}$ at or below the cutoff value were classified as the low-MIB-1 LI group. All other tumors, with the MIB-1 LI values above the cutoff value, formed the high-MIB-1 LI group.

Among these parameters, the high MIB-1 LIs of the fibroblasts forming FF and FF fibrosis Grade 3 showed significant correlations with LNM in the univariate analyses, and both of them significantly increased the RRs of LNM in the multivariate analysis (Table 4). There were no significant associations between LNM and the MIB-1 LIs of the tumor cells, the LIs of the fibroblasts not forming FF, the MVCs, invasive tumor size, and FF diameter in the univariate analyses $(P=.222, P=.449, P=.136, P$ $=.085$, and $P=.443$, respectively).

The multivariate analysis showed that the MIB-1 LIs of the fibroblasts forming FF and MVCs still significantly increased the HRs of DOM (Table 4; Fig. 6); however, nodal status failed to increase the HR of DOM in the multivariate analysis. The MIB-1 LIs of the tumor cells, the LIs of the fibroblasts not forming FF, FF fibrosis grade, FF diameter, invasive tumor size, and adjuvant therapy status were not significantly correlated with DOM in the univariate analyses $(P=.064, P=.559, P=.964, P=.135, P=$ .104 , and $P=.205$, respectively).

In the univariate analyses, there were significant associations between mortality rate (MR) and the MIB-1 LIs of the tumor cells, the MVCs, and FF diameter $(P=.004, P=.030$, and $P=.0266$, respectively). However only the MIB-1 LIs of the tumor cells still significantly increased the HR of MR in the multivariate analysis (Table 4). The MVCs and FF diameter failed to significantly increase the HRs of MR. There were no significant correlations between MR and the MIB-1 LIs of the fibroblasts forming and not forming FF, FF fibrosis grade, invasive tumor size, or adjuvant therapy status in the univariate analyses $(P=.173, P=.571, P=.750, P$ $=.351$, and $P=.237$, respectively).

LNM was observed in 38 patients with IDCs without FF. Only five patients had DOM, and only four 
TABLE 2. Pearson's Correlation between the MIB-1 LIs of Tumor Cells and of Fibroblasts and the Microvessel Counts in IDCs with FF

\begin{tabular}{|c|c|c|c|c|}
\hline \multirow[b]{2}{*}{ Parameters } & \multicolumn{3}{|c|}{ MIB-LI (\%) } & \multirow{2}{*}{$\begin{array}{c}\text { Microvessel Count } \mathrm{r} \text {, } \\
(P)\end{array}$} \\
\hline & $\begin{array}{l}\text { Tumor Cell } \mathrm{r} \text {, } \\
(P)\end{array}$ & $\begin{array}{l}\text { Fibroblast, Fibrotic } \\
\text { Focus } \mathrm{r},(P)\end{array}$ & $\begin{array}{c}\text { Fibroblast, Non-Fibrotic } \\
\text { Focus r, }(P)\end{array}$ & \\
\hline \multicolumn{5}{|l|}{ IDC with FF ( $n=105)$} \\
\hline T, MIB-1 LI & - & $0.45(<.01)$ & $0.19(.04)$ & $0.13(.20)$ \\
\hline F, FF MIB-1 LI & $0.45(.01)$ & - & $0.41(<.01)$ & $0.08(.41)$ \\
\hline F, non-FF MIB-1 LI & $0.19(.04)$ & $0.41(<.01)$ & - & $0.20(.04)$ \\
\hline FF diameter (mm) & $0.15(.12)$ & $0.15(.14)$ & $0.03(.75)$ & $0.01(.01)$ \\
\hline FF grade $(1,2,3)$ & $-0.33(<.01)$ & $-0.03(.78)$ & $-0.11(.26)$ & $0.20(.04)$ \\
\hline
\end{tabular}

FF, fibrotic focus; LI, labeling index; T, tumor cell; F, fibroblast.

TABLE 3. Parameters Significantly Associated with the Presence of Fibrotic Focus

\begin{tabular}{|c|c|c|c|c|c|}
\hline \multirow{2}{*}{ Parameters } & \multirow{2}{*}{ Total } & \multicolumn{4}{|c|}{ Multivariate for FF } \\
\hline & & FF (\%) & $\mathrm{RR}$ & $95 \% \mathrm{CI}$ & $P$ value \\
\hline All cases & 204 & $105(51)$ & & & \\
\hline \multicolumn{6}{|l|}{ MIB-1 LI (\%) of tumor cells } \\
\hline Low & 105 & $39(37)$ & Referent & & \\
\hline High & 99 & $66(67)$ & 3.2 & $1.6-6.1$ & $<.001$ \\
\hline \multicolumn{6}{|l|}{ Invasive tumor size (mm) } \\
\hline$\leq 20$ & 74 & $24(32)$ & Referent & & \\
\hline$>20$ & 130 & $81(62)$ & 2.2 & $1.2-4.4$ & .017 \\
\hline \multicolumn{6}{|l|}{ Lymphatic invasion } \\
\hline Absent & 106 & $41(39)$ & Referent & & \\
\hline Present & 90 & $63(70)$ & 3.9 & $1.7-6.1$ & $<.001$ \\
\hline Nodal status & 196 & 102 & & & \\
\hline Absent & 92 & $36(39)$ & Referent & & \\
\hline Positive & 104 & $66(63)$ & 2.0 & $1.1-3.7$ & .032 \\
\hline \multicolumn{6}{|l|}{ MVC } \\
\hline Low & 106 & $46(43)$ & Referent & & \\
\hline High & 98 & $59(60)$ & 2.0 & $1.0-3.7$ & .042 \\
\hline \multicolumn{6}{|l|}{ Adipose tissue invasion } \\
\hline Absent & 21 & $4(19)$ & Referent & & \\
\hline Present & 183 & $101(56)$ & & & \\
\hline \multicolumn{6}{|l|}{ Tumor necrosis } \\
\hline Absent & 162 & $76(47)$ & Referent & & \\
\hline Present & 42 & $29(69)$ & & & \\
\hline \multicolumn{6}{|l|}{ Vascular invasion } \\
\hline Absent & 154 & $70(45)$ & Referent & & \\
\hline Present & 50 & $35(70)$ & & & \\
\hline \multicolumn{6}{|l|}{ Histological grade } \\
\hline 1 and 2 & 142 & $65(46)$ & Referent & & \\
\hline 3 & 62 & $40(65)$ & & & \\
\hline \multicolumn{6}{|l|}{ ER status } \\
\hline Negative & 59 & $37(63)$ & Referent & & \\
\hline Positive & 145 & $68(47)$ & & & \\
\hline
\end{tabular}

The multivariate analysis for the presence of FF was performed by the logistic regression model and adjusted with parameters significantly correlated with the presence of fibrotic focus by using the step-down method until all the remaining factors were significant at a $P$ level below .05. FF, fibrotic focus; $\mathrm{RR}$, relative risk; CI, confidence interval; LI, labeling index; MVC, microvessel count.

died of the disease. Because there was no significant difference between the MIB-1 LIs of the fibroblasts in the inner and outer area, the MIB-1 LIs of the fibroblasts in the inner area were used to assess LNM, DOM, and OS in IDCs without FF. Among the MIB-1 LIs of the tumor cells, the LIs of the fibroblasts, and the MVCs, only the MIB-1 LIs of the fibroblasts were significantly correlated with LNM $(P=.007)$. The MIB-1 LIs of the tumor cells and MVCs were not significantly correlated with LNM ( $P$ $=.333$ and $P=.883$, respectively). In addition, there were significant association between the MIB-1 LIs of the fibroblasts and DOM and OS ( $P=$ .029 and $P=.012$, respectively). The MIB-1 LIs of the tumor cells and MVCs were not significantly correlated with DOM and OS $(P=.653$ and .344 , and $P=.056$ and .094 , respectively).

\section{DISCUSSION}

Breast cancer with FF-like lesions was previously reported as cancer with sclerotic foci by Linell et al. (16), and as scar cancer by Fisher et al. (17). Fisher et al. (17) divided scar cancers into five subtypes according to the histological features of the sclerotic foci, and FF appears to be similar to Type 2 or Type 3 in their classification. It has been reported 
TABLE 4. Multivariate Analyses for LNM, DOM, and OS in IDC with FF

\begin{tabular}{|c|c|c|c|c|c|}
\hline \multirow{2}{*}{ Parameters } & \multirow{2}{*}{ Total } & \multicolumn{4}{|c|}{ Multivariate for LNM } \\
\hline & & $\mathrm{LNM}+(\%)$ & RR & $95 \% \mathrm{CI}$ & $P$ value \\
\hline IDCs with FF & 102 & $66(65)$ & & & \\
\hline \multicolumn{6}{|l|}{ MIB-1 LI (\%) of fibroblasts forming FF } \\
\hline Low & 54 & $25(46)$ & Referent & & \\
\hline High & 48 & $41(85)$ & 8.9 & $3.1-25.2$ & $<.001$ \\
\hline \multicolumn{6}{|l|}{ Fibrosis grade of FF } \\
\hline 1 and 2 & 44 & $23(52)$ & Referent & & \\
\hline \multirow[t]{3}{*}{3} & 58 & $43(74)$ & 3.7 & $1.4-9.8$ & .011 \\
\hline & & \multicolumn{4}{|c|}{ Multivariate for DOM } \\
\hline & & DOMR (\%) & HR & $95 \%$ CI & $P$ value \\
\hline IDCs with FF & 105 & $24(23)$ & & & \\
\hline \multicolumn{6}{|l|}{ MIB-1 LI (\%) of fibroblasts forming FF } \\
\hline Low & 57 & $9(16)$ & Referent & & \\
\hline High & 48 & $15(31)$ & 3.1 & $1.3-7.2$ & .009 \\
\hline \multicolumn{6}{|l|}{ MVC } \\
\hline Low & 55 & $9(16)$ & Referent & & \\
\hline High & 50 & $15(30)$ & 2.6 & $1.1-6.0$ & .030 \\
\hline \multicolumn{6}{|l|}{ Nodal status } \\
\hline Absent & 36 & $4(11)$ & Referent & & \\
\hline \multirow[t]{3}{*}{ Present } & 66 & $19(29)$ & & & \\
\hline & & \multicolumn{4}{|c|}{ Multivariate for OS } \\
\hline & & $\begin{array}{l}\text { MR } \\
(\%)\end{array}$ & HR & $95 \%$ CI & $P$ value \\
\hline IDCs with FF & 105 & $18(17)$ & & & \\
\hline \multicolumn{6}{|l|}{ MIB-1 LI (\%) of tumor cells } \\
\hline Low & 57 & $5(9)$ & Referent & & \\
\hline High & 48 & $13(27)$ & 4.7 & $1.7-13.7$ & .045 \\
\hline \multicolumn{6}{|l|}{ MVC } \\
\hline Low & 55 & $6(10)$ & Referent & & \\
\hline High & 50 & $12(24)$ & & & \\
\hline \multicolumn{6}{|l|}{ Nodal status } \\
\hline Absent & 36 & $3(8)$ & Referent & & \\
\hline Present & 66 & $15(22)$ & & & \\
\hline \multicolumn{6}{|l|}{ Diameter of FF (mm) } \\
\hline$\leq 10$ & 62 & $7(11)$ & Referent & & \\
\hline$>10$ & 43 & $11(26)$ & & & \\
\hline
\end{tabular}

In order to identify the parameter with the strongest effect on LNM, DOM, and OS in IDCs with FF, multivariate analyses were performed for parameters significantly correlated with the presence of FF in the multivariate analysis, fibrosis grade and diameter of FF. The multivariate analyses for LNM were performed by the logistic regression model, and those for DOM and MR were performed by the Cox proportional hazard regression model. The multivariate analyses were adjusted with parameters that were significantly correlated with LNM, DOM, or OS listed on the table using the step-down method until all the remaining factors were significant at a $P$ level below .05 .

OS, overall survival; FF, fibrotic focus; RR, relative risk; CI, confidence interval; LNM, lymph node metastasis; LNM+, positive nodal metastasis rate; DOM, distant organ metastasis; DOMR, DOM rate; MR, mortality rate; IDC, invasive ductal carcinoma; HR, hazard rate; LI, labeling index; MVC, microvessel count.

that the survival time of patients with Types 2 and 3 scar cancers is not significantly different from nonscar cancer (17). Scar cancer has also been shown to have a better outcome and to be richer in steroid hormone receptors than nonscar cancers $(18,19)$. By contrast, we have already reported that the patients with IDCs with FF have significantly poorer short- and long-term survival periods than those without FF $(1,2)$. The studies on scar cancers did not classify the cases according to histological subtype and also included other histological types, such as lobular, mucinous, medullary, or squamous cell carcinoma and noninvasive ductal carcinoma, in addition to IDC (17-19). To more precisely clarify the clinicopathological significance of FF in primary breast cancer, it was necessary to study tumors of the same histological type. We investigated whether the presence of FF in IDCs, the most common histological type of breast cancer, is of prognostic significance for patient outcome, and we clearly demonstrated significant correlations between the presence of FF and LNM, DOM, and OS in the different IDC patient series in this study. These findings again confirm the prognostic significance of the presence of FF in patients with IDC and strongly suggest the presence of $\mathrm{FF}$ as a universal prognostic histologic parameter for the patients with IDC. Accordingly, IDCs should be classified into subtypes with and without FF in order to accurately evaluate patient outcome.

Multivariate analysis clearly demonstrated that IDCs with FF have significantly higher tumor cell proliferative activity, higher MVCs, larger invasive tumor size, and a higher nodal metastasis level than 


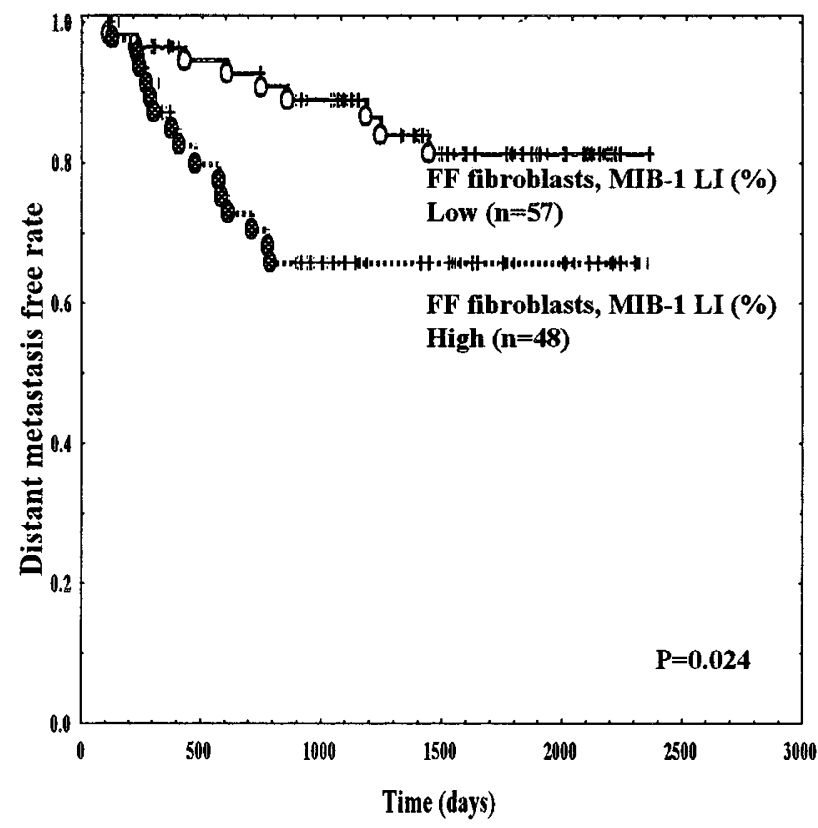

FIGURE 6. Distant-organ metastasis (DOM) curve according to the MIB-1 labeling indexes of the fibroblasts forming FF. In invasive ductal carcinomas with fibrotic focus (FF), the tumors with highly proliferative fibroblasts forming FF show a significantly shorter DOM-free survival rate than those with poorly proliferative fibroblasts forming $\mathrm{FF}$ ( $P=$ .024).

those without $\mathrm{FF}$, and these parameters have been reported to have significant effects on DOM or OS (5-7, 20-22). In addition, the FF-forming fibroblasts had the highest proliferative activity among the intratumoral fibroblasts in IDCs. We previously reported that the proliferative activity of the intratumoral fibroblasts in IDCs plays an important role in LNM or DOM (23). Accordingly, these parameters probably heighten the malignant potential of IDCs with FF and may be responsible for the differences in biological characteristics between IDCs with and without FF. In addition, the presence of FF may be a good histological parameter for estimating the degree of proliferative activity of intratumoral fibroblasts within IDCs.

This study also clearly demonstrated that the proliferative activity of the fibroblasts forming FF plays a very important role in LNM and DOM. The fibrosis grade of FF also had a significant independent association with LNM. These findings indicate that the biological characteristics of fibroblasts forming FF play an important role in tumor metastasis by IDCs with FF. The tumor-stromal interaction plays an important role in tumor invasion or metastasis $(4,24,25)$, and it has been reported that fibroblasts within a tumor express proteinase, a very important determinant of tumor cell invasion or metastasis $(26,27)$. These findings suggest that highly proliferative fibroblasts forming FF secrete larger amounts of proteinases than poorly proliferative fibroblasts forming $\mathrm{FF}$ and that this may in- crease the malignant potential of IDC with FF. Thus, evaluation of the proliferative activity of the fibroblasts forming FF will probably enable us to select IDCs with FF having the highest metastatic potential.

Although the proliferative activity of fibroblasts forming FF was the only parameter significantly associated with LNM or DOM, it had no effect on OS. The predictive power of proliferative activity of fibroblasts forming FF may be negated by other parameters having more powerful impact on OS, for example, the proliferative activity of the tumor cells, MVCs, or nodal status, because in IDCs without FF, the low-risk group, no other parameters had a stronger effect on OS than the proliferative activity of the fibroblasts within the tumor. This suggests that highly proliferative fibroblasts forming FF provide a suitable environment for tumor cell metastasis in IDCs with FF and that the proliferative activity of the fibroblasts is a useful parameter for selecting IDCs with the highest malignant potential among IDCs without FF.

The first hypothesis for the mechanism heightening the proliferative activity of intratumoral fibroblasts is that interaction between tumor cells and intratumoral fibroblasts plays an important role in forming FF. We previously demonstrated a significant correlation between fibroblast growth factor receptor expression by fibroblasts forming $\mathrm{FF}$ and basic fibroblast growth factor (bFGF) expression by tumor cells, suggesting a paracrine action of bFGF and fibroblast growth factor receptor between the tumor cells and the fibroblasts (28). Thus, tumor cells may accelerate the proliferative activity of intratumoral fibroblasts in IDCs, and highly proliferative fibroblasts may form FF.

The second hypothesis is that an autocrine mechanism of intratumoral fibroblasts themselves contributes to the formation of FF in IDCs. A significant association between the proliferative activity of the tumor cells and the fibroblasts was observed in IDCs without FF as well as in those with FF. If the formation of FF in IDCs depends only on a paracrine mechanism between tumor cells and intratumoral fibroblasts, all IDCs should have FF, but not all of them do. It has been reported that certain oncogenes can increase fibroblast proliferation $(29,30)$ and proliferative fibroblasts in fibroproliferative disease, such as scleroderma expressing c-myb proto-oncogene (31). Therefore, expression of some oncogene by fibroblasts forming FF may increase the proliferative activity of the fibroblasts themselves.

In addition to these hypotheses, proliferating intratumoral fibroblasts may deviate from the mechanism of apoptosis and form FF in IDCs $(32,33)$. If FF is formed only by a paracrine or autocrine growth mechanism of intratumoral fibroblasts, a 
significant correlation should be observed between FF diameter and the proliferative activity of the fibroblasts forming FF. However, no such correlation was found in this study. This suggests that intratumoral fibroblasts escaping from apoptosis live longer than those that do not and can produce FF in IDCs. It is, therefore, very important to clarify whether the proliferation of the intratumoral fibroblasts that form FF is dependent on the mechanism of apoptosis.

The results of this study strongly suggest that highly proliferative fibroblasts, especially forming FF, support the tumor progression in patients with IDCs. Therefore, treatments to suppress the activities of the fibroblasts may give patients with IDCs great benefit in terms of improvement of their prognosis.

Acknowledgment: We thank Miss Megumi Horino for the tissue preparation.

\section{REFERENCES}

1. Hasebe T, Tsuda H, Hirohashi S, Shimosato Y, Iwai M, Imoto $\mathrm{S}$, et al. Fibrotic focus in invasive ductal carcinoma: an indicator of high tumor aggressiveness. Jpn J Cancer Res 1996;87:385-94.

2. Hasebe T, Tsuda H, Tsubono Y, Imoto S, Mukai K. Fibrotic focus in invasive ductal carcinoma: a histopathological prognostic parameter for tumor recurrence and tumor death within three years after the initial operation. Jpn J Cancer Res 1997;88:590-9.

3. Hasebe T, Tsuda H, Hirohashi S, Shimosato Y, Tsubono Y, Yamamoto H, et al. Fibrotic focus in infiltrating ductal carcinoma of the breast: a significant histopathological prognostic parameter for predicting the long-term survival of the patients. Breast Cancer Res Treat 1998;49:195-208.

4. Jitsuiki Y, Hasebe T, Tsuda H, Imoto S, Tsubono Y, Sasaki S, et al. Optimizing microvessel counts according to tumor zone in invasive ductal carcinoma of the breast. Mod Pathol 1999;12:492-8.

5. Jansen RL, Hupperets PS, Arends JW, Joosten-Achjanie SR, Volovics A, Schouten HC, et al. MIB-1 labelling index is an independent prognostic marker in primary breast cancer. Br J Cancer 1998;78:460-5.

6. Biesterfeld S, Kluppel D, Koch R, Schneider S, Steinhagen G, Mihalcea AM, et al. Rapid and prognostically valid quantification of immunohistochemical reactions by immunohistometry of the most positive tumour focus. A prospective follow-up study on breast cancer using antibodies against MIB-1, PCNA, ER, and PR. J Pathol 1998;185:25-31.

7. Kumar S, Ghellal A, Li C, Byrne G, Haboubi N, Wang JM, et al. Breast carcinoma: vascular density determined using CD105 antibody correlates with tumor prognosis. Cancer Res 1999;15:856-61.

8. Sobin LH, Wittekind Ch. In: TNM Classification of malignant tumors. 5th ed. New York: Wiley-Liss; 1997. p. 93.

9. World Health Organization. In: Histological typing of breast tumors: international histological classification of tumors. 2nd ed. Geneva, Switzerland: World Health Organization; 1981. p. 20-2.

10. Elston CW, Ellis IO. Pathological prognostic factors in breast cancer. I. The value of histological grade in breast cancer: experience from a large study with long-term follow-up. Histopathology 1991;19:403-10.

11. Hsu SM, Raine L, Fanger H. The use of avidin-biotinperoxidase complex (ABC) in immunoperoxidase technique: a comparison between $\mathrm{ABC}$ and unlabeled antibody (PAP) procedures. J Histochem Cytochem 1981;29:577-80.

12. Shi SR, Key ME, Kalra KL. Antigen retrieval in formalin-fixed, paraffin-embedded tissues: an enhancement method for immunohistochemical staining based on microwave oven heating of tissue sections. J Histochem Cytochem 1991;39: 741-8.

13. Hollingsworth $\mathrm{CH}$, Kohn EC, Steinberg SM, Rothenberg ML, Merino MJ. Tumor angiogenesis in advanced stage ovarian carcinoma. Am J Pathol 1995;147:33-41.

14. Truett J, Cornfield J, Kannel W. A multivariate analysis of the risk of coronary heart disease in Framingham. J Chronic Dis 1967;20:511-24.

15. Cox DR. Regression models and life-tables. J Roy Stat Soc 1972;34:187-220.

16. Linell F, Ljungberg O, Andersson I. Breast carcinoma: aspects of early stages, progression, and related problems. Acta Pathol Microbiol Scand Suppl 1980;272:1-233.

17. Fisher ER, Palekar AS, Sass R, Fisher B. Scar cancers: pathologic findings from the National Surgical Adjuvant Breast Project (protocol no. 4)-IX. Breast Cancer Res Treat 1983;3: 39-59.

18. Toikkanen S, Joensuu H. Long-term prognosis of scar and non-scar cancers of the breast. APMIS 1990;98:1033-8.

19. Partanen S, Hyvarinen H. Scar and non-scar ductal cancer of the female breast. Observations on patient age, tumour size, and hormone receptors. Virchows Arch A Pathol Anat Histopathol 1987;412:145-9.

20. Fisher B, Bauer M, Wickerham L, Redmond CK, Fisher ER. Relation of number of positive axillary nodes to the prognosis of patients with primary breast cancer. Cancer 1983;52: 1551-7.

21. Carter CL, Allen C, Henson DE. Relation of tumor size, lymph node status and survival in 24,740 breast cancer cases. Cancer 1989;63:181-7.

22. Wolman SR, Feiner HD, Schinella RA. A retrospective analysis of breast cancer based on outcome differences. Hum Pathol 1991;22:475-80.

23. Hasebe T, Sasaki S, Imoto S, Ochiai A. Proliferative activity of intratumoral fibroblasts is closely correlated with lymph node and distant organ metastasis of invasive ductal carcinoma of the breast. Am J Pathol 2000;156:1701-10.

24. Booth C, Harnden P, Trejdosiewicz LK, Scriven S, Selby PJ, Southgate J. Stromal and vascular invasion in a human in vitro bladder cancer model. Lab Invest 1997;76:843-57.

25. Uria JA, Stahle-Backdahl M, Seiki M, Fueyo A, Fueyo A, Lopez-Otin C. Regulation of collagenase-3 expression in human breast carcinomas is mediated by stromal-epithelial cell interactions. Cancer Res 1997;57:4882-8.

26. Noel A, Hajitou A, L’Hoir C, Maquoi E, Baramova E, Lewalle $\mathrm{JM}$, et al. Inhibition of stromal matrix metalloproteases: effects on breast tumor promotion by fibroblasts. Int J Cancer 1998;76:267-73.

27. Afzal S, Lalani EN, Poulsom R, Stubbs A, Rowlinson G, Sato $\mathrm{H}$, et al. MT1-MMP and MMP-2 mRNA expression in human ovarian tumors: possible implications for the role of desmoplastic fibroblasts. Hum Pathol 1998;29:155-65.

28. Hasebe T, Imoto S, Ogura T, Mukai K. Significance of basic fibroblast growth factor and fibroblast growth factor receptor protein expression in the formation of fibrotic focus in invasive ductal carcinoma of the breast. Jpn J Cancer Res 1997;88:877-85.

29. Yan Y, Ouellette MM, Shay JW, Wright WE. Age-dependent 
alterations of c-fos and growth regulation in human fibroblasts expressing the HPV16 E6 protein. Mol Biol Cell 1996; 7:975-83.

30. Orlandini M, Semplici F, Ferruzzi R, Meggio F, Pinna LA, Oliviero S. Protein kinase CK2alpha' is induced by serum as a delayed early gene and cooperates with Ha-ras in fibroblast transformation. J Biol Chem 1998;273:21291-7.

31. Piccinini G, Luchetti MM, Caniglia ML, Carossino AM, Montroni $\mathrm{M}$, Introna $\mathrm{M}$, et al. c-myb proto-oncogene is expressed by quiescent scleroderma fibroblasts and, unlike B-myb gene, does not correlate with proliferation. J Invest Dermatol 1996;106:1281-6.

32. Pablos JL, Carreira PE, Serrano L, Del Castillo P, GomezReino JJ. Apoptosis and proliferation of fibroblasts during postnatal skin development and scleroderma in the tightskin mouse. J Histochem Cytochem 1997;45:711-9.

33. Guinee D Jr, Brambilla E, Fleming M, Hayashi T, Rahn M, Koss M, et al. The potential role of BAX and BCL-2 expression in diffuse alveolar damage. Am J Pathol 1997;151:9991007.

\section{Book Review}

\section{Henderson JE, Goltzman D, editors: The Osteo- porosis Primer, 388 pp, Cambridge, Cam- bridge University Press, 2000 (\$64.95).}

Although there are several books on metabolic bone disease, The Osteoporosis Primer is an excellent introductory text, most useful to those interested in a more general approach to the field. It is divided into four sections dealing with the molecular and cellular environment of the bone, determinants of peak bone mass, pathophysiology of the aging skeleton, and clinical aspects of osteoporosis. Throughout the text, clinical aspects of the disease are constantly correlated with the underlying cellular and molecular phenomena. Informative tables and lucid illustrations make the key concepts easy to understand and the book easy to read. It is also a very good source of references for further reading. My only suggestion for the next edition would be to include more on the histomorphometric analysis of osteoporotic bone. Microscopic morphometry has been widely used for evaluating osteoporosis, and such studies have contributed to the better understanding of pathophysiologic mechanisms, and for evaluating the effectiveness of new therapeutic approaches.

This book is recommended primarily to general internists, family physicians, and gerontologists, but it would be of interest to orthopedic surgeons, gynecologists, and endocrinologists as well. Pathologists might particularly value a comprehensive description of cellular and subcellular aspects of metabolic bone disorders.

Ivan Kresimir Lukic

University of Zagreb School of Medicine

Zagreb, Croatia 